Letter to the Editor

\title{
Teaching professionalism and professional ethics using the hidden curriculum
}

\author{
Soheyla Kalantari ${ }^{1}$, Ghorban Mohammad Koochaki ${ }^{1}$, Leila Jouybari ${ }^{2 *}$ Akram Sanagoo ${ }^{2}$, Aliakbar Aghaie \\ Nejad $^{1}$
}

(Received: 27 Dec 2015 ; Accepted: 26 Apr 2016)

We are writing this letter to draw the attention of the faculty members of medical universities to the formation of professional ethics and professionalism in the clinical and theoretical education of nursing and operating room students using the hidden curriculum.

Professional values are standards for clinical practice, which are based on the agreement of professional groups and experts. These values are adopted in providing a framework to evaluate the ideas and perspectives that influence behavior (1). Clinical competence, adequate skills for medical practice, keeping updated, honesty, confidentiality and appropriate communication with patients are among the most imperative professional values (2).

To develop professionalism, medical education in Iran has undergone several integrative reforms by the Ministry of Health and Medical Education, as part of the health system reform (stage four). The comprehensive program of higher education in the Iranian health system is a strategic plan developed based on key documents, such as Iran's Vision 2025, Comprehensive Scientific Map of Iran, Iran's Comprehensive Health Sector Road Map, and Health System Reform. One of the macropolicies of this evolutionary reform plan is the institutionalization of professional ethics in universities (3).

Hidden curriculum refers to the process of cultural conduction and transmission of moral norms in relation to the feelings and behaviors of medical students in clinical settings as an ethical environment. In other words, a hidden curriculum consists of the implicit norms, values and beliefs that are transferred through rules and regulations, structures, routines, and social interactions in educational institutions. While only a part of this culture is transmitted via formal education, values, beliefs and accepted behaviors are passed on to students through the hidden curriculum (4).

In general, learning experiences that are resulted from the hidden curriculum encompass a set of expectations and values without theoretical knowledge. Therefore, hidden curriculum could remarkably influence professional practice in the clinical environment.

In theoretical education, medical, nursing and paramedical students only receive tutoring on disease management and formal communication with patients and clients. However, in clinical settings, they are faced with actual cases, complicated demands, chronic patients with different socioeconomic status, and interactions with other healthcare providers and educators, which might be extremely challenging or confusing (5).

Hidden curriculum plays a pivotal role in conveying the required knowledge and clinical skills to deal with actual patients, physicians, diseases and reality of the society (6). Evidence suggests that the hidden curriculum might occasionally be contrary to the learned clinical knowledge of students. Meanwhile, the ethical aspect of hidden curriculum could appear as a barrier against proper decisionmaking under different circumstances (6).

Current data in this regard indicates that through

\footnotetext{
${ }^{1}$ Department of Operating Room, Paramedical School, Golestan University of Medical Sciences, Gorgan, Iran

2,* Corresponding author: Department of Nursing, Education Development Office, Golestan University of Medical Sciences, Gorgan, Iran.

Email: jouybari@goums.ac.ir
} 
the proper management of hidden curriculum, it is possible to predict and improve qualified behaviors and professionalism in the clinical environment (1, 7-10). Domestic studies on the impact of the hidden curriculum on professionalism have mainly adopted qualitative approaches in order to assess the experiences of students (11).

Authors of this letter have worthy experiences regarding the application of narrative writing and reflective journaling about clinical errors and ethical events by nursing students during internship in the hospital $(12,13)$, in addition to individual and group reflective journaling by operating room students and their educators in clinical practice with the propose of developing professionalism.

In conclusion, a beneficial approach to teach ethics through the hidden curriculum involves opportunities for students to have open dialogue, critical discussions, and brainstorming with clinical educators. Moreover, they must attempt to practice reflective journaling and narrative writing about ethical values, professional malpractice, and professional behaviors in clinical environments.

\section{References}

1. Parvan K, Hosseini F, Zamanzadeh V. Professional values from nursing students' perspective in Tabriz University of medical sciences: a pilot study. Iran J Nurs 2012; 25(76):28-41.

2. Allami A, Shirazi M. Faculty members experience about teaching of medical professionalism in Qazvin University of medical sciences. J Res Med Educ 2015; 7(2):1-11.

3. Asch DA, Weinstein DF. Innovation in medical education. N Engl J Med 2014; 371(9):794-5.

4. Chuang AW, Nuthalapaty FS, Casey PM, Kaczmarczyk JM, Cullimore AJ, Dalrymple JL, et al. To the point: reviews in medical education taking control of the hidden curriculum.
Am J Obstet Gynecol 2010; 203(4):316.e1-6.

5. Arnold RM. Formal, informal, and hidden curriculum in the clinical years: where is the problem? J Palliat Med 2007; 10(3):646-8.

6. GoftonW, Regehr G. What we don't know we are teaching: unveiling the hidden curriculum. Clin Orthop Relat Res 2006; 449:20-7.

7. Gaufberg EH, Batalden M, Sands R, Bell SK. The hidden curriculum: what can we learn from third-year medical student narrative reflections? Acad Med 2010; 85(11):1709-16.

8. Mosalanejad L, Parandavar N, Rezaie E. Students' experience about the hidden curriculum: a qualitative study. J Rafsanjan Univ Med Sci 2014; 13(2):111-24 (Persian).

9. Yamani N, Liaghatdar MJ, Changiz T, Adibi P. How do medical students learn professionalism during clinical education? A qualitative study of faculty members' and interns' experiences. Iran J Med Educ 2010; 9(4):382-95 (Persian).

10. Rogers DA, Boehler ML, Roberts NK, Johnson V. Using the hidden curriculum to teach professionalism during the surgery clerkship. J Surg Educ 2012; 69(3):423-7.

11. Karimi Z, Ashktorab T, Mohammadi E, Abedi HA. Using the hidden curriculum to teach professionalism in nursing students. Iran Red Crescent Med J 2014; 16(3):e15532.

12. Jouybari L, Sanagoo A. The use of "reflection" as a tool to foster critical thinking of nursing students based on religious teachings. Awarded educational scholarship, The 4th Shahid Motahari National Festival. 2011. Accessed from: http:// aac.behdasht.gov.ir/uploads/179_331_BestFarayand4.pdf (Access date: Dec 2015) (Persian).

13. Jouybari L, Sanagoo A. The use of clinical narratives as a tool for learning and teaching inspired by the verse "therefore, take heed you that have eyes". Awarded educational scholarship, The 5th Shahid Motahari Festival. 2012. Accessed from: http://www.goums.ac.ir/page. php?slct_pg_id=3589\&sid=16\&slc_lang=fa (Access date: Dec 2015) (Persian). 